Sensors 2011, 11, 3765-3779; doi:10.3390/s110403765

Article

\title{
Hyperspectral and Chlorophyll Fluorescence Imaging to Analyse the Impact of Fusarium culmorum on the Photosynthetic Integrity of Infected Wheat Ears
}

\section{Elke Bauriegel $^{1}{ }^{1}$, Antje Giebel ${ }^{1}$ and Werner B. Herppich ${ }^{2}$}

1 Department of Engineering for Crop Production, Leibniz-Institute for Agricultural Engineering Potsdam-Bornim, D-14469 Potsdam, Germany; E-Mail: agiebel@atb-potsdam.de

2 Department of Horticultural Engineering, Leibniz-Institute for Agricultural Engineering Potsdam-Bornim, D-14469 Potsdam, Germany; E-Mail: wherppich@atb-potsdam.de

* Author to whom correspondence should be addressed; E-Mail: ebauriegel@atb-potsdam.de; Tel.: +49-331-5699-414; Fax: +49-331-5699-849.

Received: 24 January 2011; in revised form: 23 March 2011 / Accepted: 25 March 2011 / Published: 28 March 2011

\begin{abstract}
Head blight on wheat, caused by Fusarium spp., is a serious problem for both farmers and food production due to the concomitant production of highly toxic mycotoxins in infected cereals. For selective mycotoxin analyses, information about the on-field status of infestation would be helpful. Early symptom detection directly on ears, together with the corresponding geographic position, would be important for selective harvesting. Hence, the capabilities of various digital imaging methods to detect head blight disease on winter wheat were tested. Time series of images of healthy and artificially Fusarium-infected ears were recorded with a laboratory hyperspectral imaging system (wavelength range: $400 \mathrm{~nm}$ to $1,000 \mathrm{~nm}$ ). Disease-specific spectral signatures were evaluated with an imaging software. Applying the 'Spectral Angle Mapper' method, healthy and infected ear tissue could be clearly classified. Simultaneously, chlorophyll fluorescence imaging of healthy and infected ears, and visual rating of the severity of disease was performed. Between six and eleven days after artificial inoculation, photosynthetic efficiency of infected compared to healthy ears decreased. The severity of disease highly correlated with photosynthetic efficiency. Above an infection limit of 5\% severity of disease, chlorophyll fluorescence imaging reliably recognised infected ears. With this technique, differentiation of the severity of disease was successful in steps of $10 \%$. Depending on the quality of chosen regions of interests, hyperspectral imaging readily detects head blight $7 \mathrm{~d}$ after inoculation
\end{abstract}


up to a severity of disease of 50\%. After beginning of ripening, healthy and diseased ears were hardly distinguishable with the evaluated methods.

Keywords: chlorophyll defect; fungal diseases; non-destructive; non-invasive sensor application; potential maximum photochemical efficiency of PSII $\left(\mathrm{F}_{\mathrm{v}} / \mathrm{F}_{\mathrm{m}}\right)$; Triticum aestivum L. 'Taifun'

\section{Introduction}

Many Fusarium spp. may cause serious grain contamination with mycotoxins (trichothecenes), mainly deoxynivalenol (DON) and 3-acetyl DON (3-ADON), on wheat [1]. Up to now, true infection of ears can only be determined with time consuming and expensive pre- and postharvest laboratory tests (serological rapid tests, Fast-DON-ELISA-test, counting methods; [2]). Thus, the ability to identify infections in the field could prevent exploitation of contaminated grain lots, finally, reducing toxin-burden of food- and feedstuffs.

Head blight, like other fungal and viral infections, is characterised by a complete destruction of the cellular integrity of the impacted tissues leading to cell death and degradation of chlorophylls. Damage is mostly accompanied by a transient increase in transpiration, followed by tissue desiccation. The resulting variation of tissue temperature has been successfully detected by thermal imaging, at least under controlled conditions [3]. On the other hand, chlorophyll degradation by means of spectral analysis in the visible range [4] allows detection of infected plant parts [5,6]. Application of spectral indices such as Normalized Difference Vegetation Index (NDVI), which has been shown to rapidly indicate plant stress [7], may provide a good discrimination between healthy and infected plant parts [8]. Including the NIR range in spectral analyses may increase the efficiency of discrimination because this wavelength range additionally includes information about tissue water content $[9,10]$.

Providing further knowledge about local centres of infections, imaging methods are especially suitable for rapid and non-invasive identification of the effective stage of plants disease in the field. In this context, spectral imaging was used to diagnose viral infections [11] and to identify spatially variable physiological processes of leaves [12]. Hyperspectral analyses have been applied to detect fungal-based grain crop diseases [13-15]. Furthermore, by the combination of both hyperspectral reflection measurements and chlorophyll fluorescence analyses (CFA) the discrimination of yellow rust on winter wheat could have been improved to an accuracy of 94-95\% [5].

CFA does not only measure the externally visible effects of infection-induced chlorophyll breakdown; it also provides comprehensive insight into potential and actual photosynthetic activity [16,17]. Photosynthesis is often considerably affected by both biotic and abiotic stresses at very early stages [18,19]. In addition, CFA imaging has already been universally applied, for instance for evaluation of the vitality of plant tissues [20,21], diseases like leaf rust and powdery mildew on cereals [22] or the infection by the tobacco mosaic virus [23].

For the detection of head blight, both methods have been shown to have great potential [24,25]. In this study, both imaging methods were applied in parallel to comprehensively analyse the respective ability of early detection of head blight disease in winter wheat ears both under laboratory and field 
conditions. Additional aims were: (1) to determine the highest possible accuracy of the detection of Fusarium infection; (2) to characterise the exact timeframe for meaningful head blight detection and (3) to determine the effect of the level of infection on detection accuracy.

\section{Experimental Section}

\subsection{Materials and Experimental Design}

Sixteen wheat caryopses (Triticum aestivum L. 'Taifun') were sown in eight pots $(0.18 \mathrm{~m} \times 0.18 \mathrm{~m})$. From these, four pots were used for controls and four for infected samples, respectively. After germination, plants were cultivated in a greenhouse. After the start of flowering, plants were inoculated with a water suspension of Fusarium culmorum spores at a concentration of 250,000 spores per $\mathrm{mL}$ on three successive days. To guarantee the spread of germs, plants were kept at $20 \pm 2{ }^{\circ} \mathrm{C}$, high humidity (70\%) and an illumination period of $12 \mathrm{~h}$ (high-pressure sodium-vapour lamps, SON-T Plus 400 W, Philips GmbH, Hamburg, Germany). Measurements started immediately after inoculation.

Developmental stages of ears were always graded according to the $\mathrm{BBCH}$ scale [26], which empirically describes plant development from dry seeds $(\mathrm{BBCH} 00)$ until the harvest product (BBCH 99). In the laboratory, plant infection levels were visually rated three times a week. Using sample pictures of infected ears, percentage infection of blighted spikelets per ear was estimated following [27]. Estimation of severity of disease occurred in distinct steps of 1\%, 2\%, 3\%, 5\% of damage at low infection levels and in 10\%-steps at higher severity. All measurements were performed on intact plants. As a unit of visual rating of head blight disease pattern, the "severity of disease" (sod) was defined. During the course of this study three independent experiments were performed to comprehensively analyse both disease development and disease recognition accuracy.

\subsection{Chlorophyll Fluorescence Imaging}

Chlorophyll fluorescence imaging was performed with a modular system (FluorCAM 700MF, PSI, Brno, Czech Republic) measuring sequences of fluorescence images with a user-defined timing of set points, measurement intervals and irradiance $[21,28,29]$. Basic fluorescence $F_{0}$ was induced by two panels of super-bright orange light emitting diodes $\left(\lambda_{\max }=620 \mathrm{~nm}, 345\right.$ LED per panel; approx. $\left.3 \mu \mathrm{mol} \mathrm{m} \mathrm{m}^{-2}\right)$. Maximum fluorescence $\left(\mathrm{F}_{\mathrm{m}}\right)$ was triggered by short-term $(1 \mathrm{~s})$ saturation light pulses (max. 2,500 $\mu \mathrm{mol}$ photons $\mathrm{m}^{-2} \mathrm{~s}^{-1}$ ) generated by an electronic shutter-equipped halogen lamp $(250 \mathrm{~W})$.

The ratio of variable fluorescence $\left(F_{v}=F_{m}-F_{0}\right)$ to maximum fluorescence, $F_{v} / F_{m}$, is an indicator of the potential maximum photochemical efficiency of photosystem II. It ranges between 0 (chlorophyll-less, dead plants) and 0.84 for healthy, intact plant parts under optimal conditions [17]. $F_{v} / F_{m}$ is well-known as a valuable tool to determine both capacity and stability of photosynthesis [29,30]. A CCD camera with a F1.2/2.8-6 mm objective and a filter system (high pass $695 \mathrm{~nm}$, low pass $780 \mathrm{~nm}$ ) recorded fluorescence images (12-bit, $512 \times 512$ pixel; maximal frequency 50 images s${ }^{-1}$ ) synchronously with the weak, non-actinic measuring-light pulses. The system was controlled by the FluorCam software (PSI, Brno, Czech Republic). In the laboratory, measurements lasted $4 \mathrm{~s}\left(\mathrm{~F}_{0}: 3 \mathrm{~s}\right.$ duration, 4 frames recorded; $F_{m}$ : $1 \mathrm{~s}$ duration, 25 frames recorded) and were performed on plants, dark-adapted for $10 \mathrm{~min}$. Six samples of both infected and control plants were continuously recorded in time series 
experiments. In addition, fifteen plants with pronounced head blight symptoms were investigated at $\mathrm{BBCH}$ stage 75. All plants were measured from the side at a distance of $0.2 \mathrm{~m}$ between ears and CCD-camera.

In addition, chlorophyll fluorescence imaging was applied on artificially infected winter wheat plants (BBCH stage 77/79) of the cultivars 'Cardos', 'Winnetou' and 'Drifter' (all resistance class 5) directly in the field. To eliminate the effects of direct sunlight on fluorescence and to pre-darken (10 min) the plants, they were partially shielded with a paper box (approx. $0.9 \mathrm{~m} \times 0.9 \mathrm{~m} \times 0.9 \mathrm{~m}$ ) during measurements. The duration of the fluorescence measurements was reduced to $2 \mathrm{~s}$ (i.e., $1 \mathrm{~s}$ for $\mathrm{F}_{0}$ measurements and $1 \mathrm{~s}$ for $\mathrm{F}_{\mathrm{m}}$ ). In total, 50 ears of varying levels of infection were investigated. Only optimally illuminated images with clearly distinguishable ears $(n=30)$ were further analysed in this experiment.

For a further evaluation and derivation of severity of disease (sod), in the chlorophyll fluorescence images, all $\mathrm{F}_{\mathrm{v}} / \mathrm{F}_{\mathrm{m}}$-pixel values were allocated to "efficiency classes" of photosynthetic activity at steps of 0.05 . In addition, they were accumulated to a "cumulative $F_{v} / F_{m}$ " $(\%)$, starting from the lowest values $(0.00)$.

\subsection{Hyperspectral Imaging}

The laboratory hyperspectral imaging device recorded reflection spectra in a wavelength range of 400 to $1,000 \mathrm{~nm}$ with a spectral resolution of $2.5 \mathrm{~nm}$. General pixel-resolution of the camera was $1,392 \times 1,024 \mathrm{px}$; however, two pixels per axis were combined to yield an effective resolution of $696 \times 512$ px. The field of view achieved generally resulted in a spatial resolution of approx. $0.4 \mathrm{~mm}$ per pixel. The system comprised a spectrograph (ImSpector V10E, Spectral Imaging Ltd., Oulu, Finland), a 12 bit, digitally temperature-compensated b/w camera (Pixelfly qe, PCO AG, Kelheim, Germany) and an accessory rotating mirror with a micro-step motor. The hyperspectral camera stored the spectra of all pixels of an image line by line. A program, developed under LabView 8.2 (National Instruments Corporation, Austin, TX, USA), was used to control the camera system and for data pre-processing, including the black/white calibration of the spectra. For this calibration, images for the black and the white adjustment were recorded with each measurement. The b/w-balance [(sample-black)/(white-black)] was performed for the entire frame during the following conversion of the hyperspectral images into the byte stream format. Heterogeneities in the pixel response across the sensor area were generally rather low and, therefore, not compensated for. The samples were illuminated with a stabilised halogen lamp $(150 \mathrm{~W})$. In addition to the six ears per variant used for chlorophyll fluorescence analysis, further six plants were examined by hyperspectral imaging with a time lag of approximately two days during three weeks (total $\mathrm{n}_{\text {infected }}=\mathrm{n}_{\text {controls }}=12$ ). All plants were recorded from the side at a distance of $0.5 \mathrm{~m}$ between plant and camera. To avoid vibrations, the ears were fixed on a black background. Exposure time, adjusted for the respective samples, was in the range of 20 to $25 \mathrm{~ms}$; a complete record lasted 20 to $30 \mathrm{~min}$.

\subsection{Data Analysis}

The classification of diseased and healthy areas was performed with the software ENVI (Research Systems Inc., Boulder, CO, USA) by means of monitored classifications in the "Spectral Angle 
Mapper" (SAM) evaluation algorithm. SAM compares the classifying spectrum of an image with a reference spectrum. The classes are allocated according to their similarity. The distinction of two reflection spectra is described with an angle, which span between related vectors [31,32]. In this paper, a vector in a multidimensional space (512 bands) was used. In addition, SAM was chosen because it is insensitive to variations of illumination [33].

The threshold of similarity of compared spectral angles was 0.1. For this purpose, regions of interest (ROIs) were established as the bases for the classification according to the two classes, diseased (8 ROIs) and healthy (10 ROIs). In a three-band false colour image (450 nm, $550 \mathrm{~nm}$, $650 \mathrm{~nm}$ ), diseased and healthy areas could be distinguished by visual inspection, which facilitated the proper manual setting of ROIs. These 18 ROIs was used to build an endmember, applied in the further calculations. To consider the effect of plant development, single hyperspectral images were repeatedly used as training images for setting ROIs. These images were excluded from later evaluation. After classification, the relative portions of pixels per image belonging to the healthy, diseased and unclassified object classes were determined. In this context, all pixels which could not be allocated to the defined classes "healthy" and "diseased" were assigned to the class "unclassified". The proportion of unclassified pixels was calculated as the difference between $100 \%$ and the sum of healthy and diseased pixels.

\section{Results}

The development of Fusarium infection, rated as severity of disease (sod), and the relevant BBCH stage of plants at respective days after inoculation (dai) are shown for the first time-series experiment in Table 1. The first symptoms of the disease became visible at the $\mathrm{BBCH}$ stage $71 / 75$ (7 dai), first ripening symptoms developed in the $\mathrm{BBCH}$ stage 81 .

Table 1. Representative example of plant development (BBCH stage) and rated disease symptoms of head blight (severity of disease, sod, $n=6$ ).

\begin{tabular}{ccccccccccc}
\hline dai & $\mathbf{5}$ & $\mathbf{7}$ & $\mathbf{9}$ & $\mathbf{1 1}$ & $\mathbf{1 4}$ & $\mathbf{1 6}$ & $\mathbf{1 8}$ & $\mathbf{2 1}$ & $\mathbf{2 3}$ & $\mathbf{2 5}$ \\
\hline $\mathrm{BBCH}$ & $65 / 71$ & $71 / 75$ & 75 & $75 / 77$ & $77 / 79$ & $79 / 81$ & $79 / 81$ & $81 / 85$ & 85 & 89 \\
$\operatorname{sod}(\%)$ & 0 & 3 & 5 & 6 & 9 & 16 & 19 & 60 & 82 & 90 \\
\hline
\end{tabular}

\subsection{Chlorophyll Fluorescence Imaging}

Distribution analyses of chlorophyll fluorescence images of ears with a sod between 2 and 100\% revealed that, in weakly diseased ears $(2 \%)$, as in healthy ears, pixel-values of photosynthetic efficiency $\left(F_{v} / F_{m}\right)$ concentrated in classes of high efficiency [0.55-0.75; Figure 1(a)]. The pixelwise $\mathrm{F}_{\mathrm{v}} / \mathrm{F}_{\mathrm{m}}$-distribution was nearly identical in control plants and very weakly diseased ears (data not shown).

In medium infected ears $(50 \%)$, the distribution of $F_{v} / F_{m}$ broadened [Figure 1(b)] due to the co-existence of both healthy (pixel-value range $0.40-0.75$ ) and diseased tissues (pixel-value range 0.00-0.40). In strongly infected ears [Figure 1(c)], pixel-values of photosynthetic efficiency only concentrated in the low-value range $(0.00-0.20)$. 
Figure 1. Pixelwise distribution of the maximum photochemical efficiency $\left(\mathrm{F}_{\mathrm{v}} / \mathrm{F}_{\mathrm{m}}\right)$ of $(\mathbf{a})$ weakly (2\%), (b) medium (50\%) and (c) strongly infected (90\%) wheat ears.
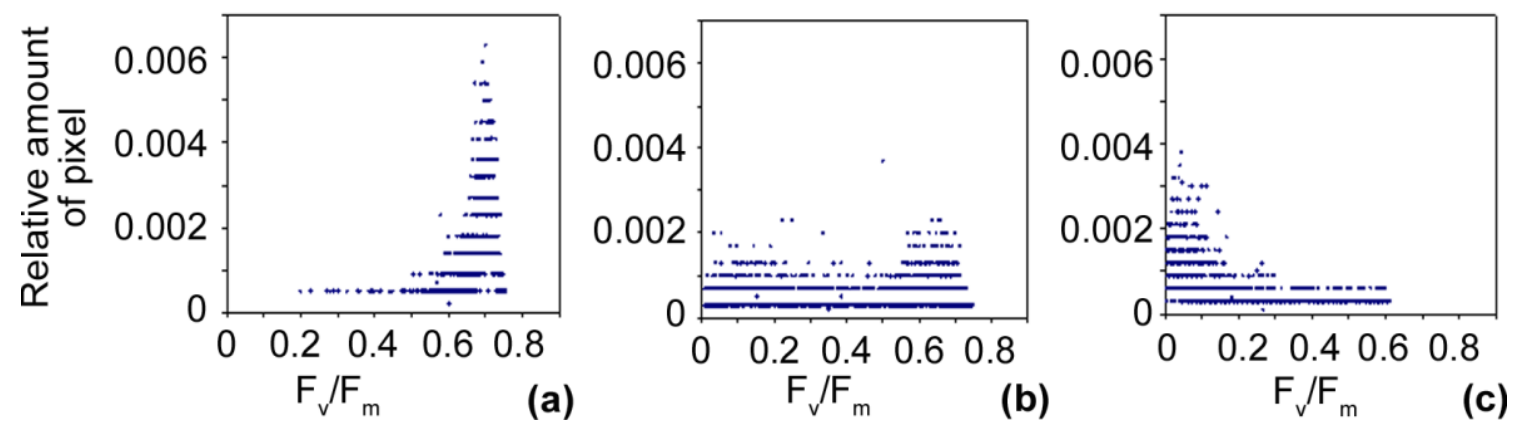

Cumulative $\mathrm{F}_{\mathrm{v}} / \mathrm{F}_{\mathrm{m}}$ appropriately characterised the head blight development of representative ears (Figure 2). At an early infection state [Figure 2(a), dai 4-6], only a few pixel values were found in low efficiency classes, while $80 \%$ of $\mathrm{F}_{\mathrm{v}} / \mathrm{F}_{\mathrm{m}}$-pixel values concentrated in high photosynthesis efficiency classes $(>0.6)$ in a healthy or weakly diseased (2-3\% infection) ear. Continuous development of infection during the course of the experiment from dai 6 to dai 22 could be easily identified by an increasing concentration of accumulated $\mathrm{F}_{\mathrm{v}} / \mathrm{F}_{\mathrm{m}}$-values in low efficiency classes. This means that moderately diseased ears (40-60\%) comprised both photosynthetically active and inactive areas. In contrast, cumulative $\mathrm{F}_{\mathrm{v}} / \mathrm{F}_{\mathrm{m}}$ obtained only low efficiency classes $(<0.3)$ if the ear was strongly diseased (90\%) $22 \mathrm{~d}$ after infection. This is also verified if the variation of average cumulative $\mathrm{F}_{\mathrm{v}} / \mathrm{F}_{\mathrm{m}}$ values of various plants of different sod is analysed [Figure 2(b)]. In this context, a cumulative $F_{v} / F_{m}$ at 0.3 seems to represent a relevant threshold to differentiate diseased and healthy ears.

Figure 2. Cumulative $\mathrm{F}_{\mathrm{v}} / \mathrm{F}_{\mathrm{m}}$-values (\%) (a) during several stages of head blight development of a single representative ear (b) Average cumulative percentage of $\mathrm{F}_{\mathrm{v}} / \mathrm{F}_{\mathrm{m}}$-values of ears at different levels of infection at dai 11.

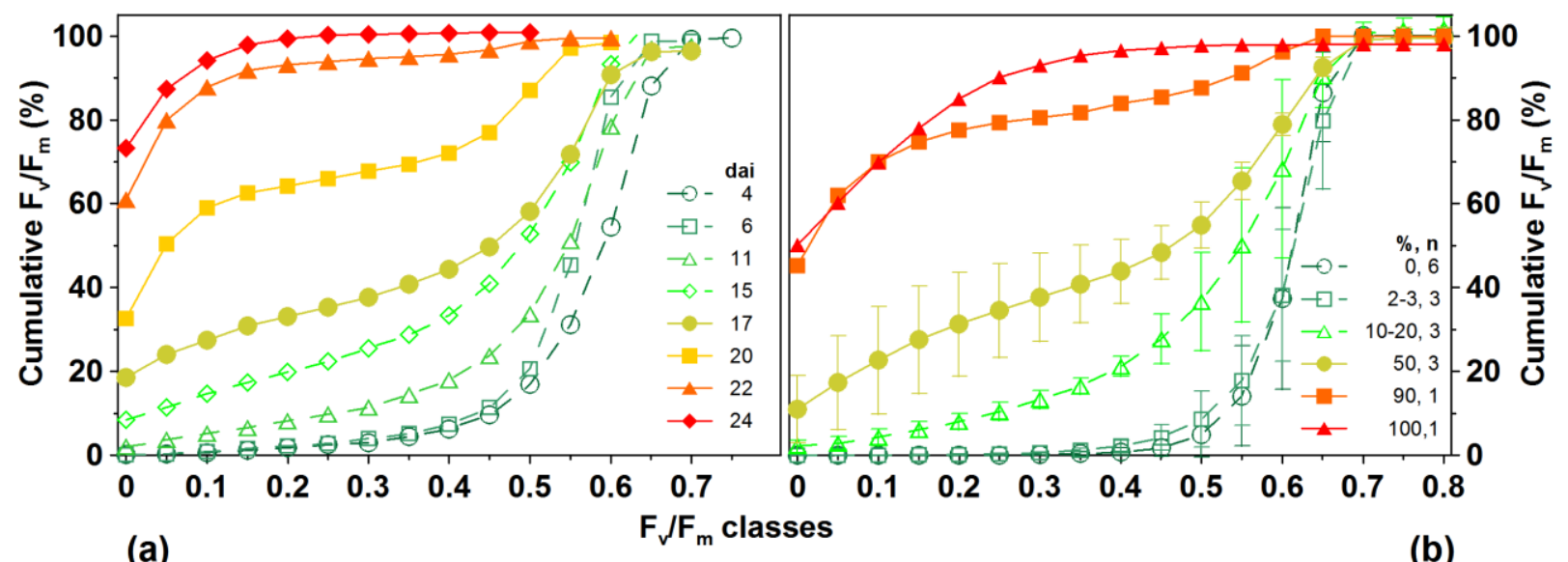

(a)

(b)

During early infection and at low infection state (sod $2 \%$ to $3 \%$ ), cumulative photosynthetic efficiency of investigated ears overlapped indicating that visual rating and CFA imaging did not obtain completely identical results [Figure 2(b)]. On the other hand, controls and clearly infected ears (sod $c a$. $5 \%$ at dai 11) could be successfully differentiated by analysing the cumulative $F_{v} / F_{m}$ classes at 0.3 
(Figure 3). Even at the first day of measurement (dai 6) the cumulative proportion of low efficiency classes was $3 \%$ higher than the control value, and rose to a median of nearly $8 \%$ within one week (dai 11). Up to a sod of $4 \%, \mathrm{~F}_{\mathrm{v}} / \mathrm{F}_{\mathrm{m}}$ of diseased and control ears did not differ. Even with a sod of $10 \%$, plants showed only minor visible symptoms of head blight one week after inoculation.

Figure 3. Cumulative $F_{v} / F_{m}$ values at 0.3 of controls (blue) and strongly (defined as $5 \%$ sod at dai 11) infected ears (red).

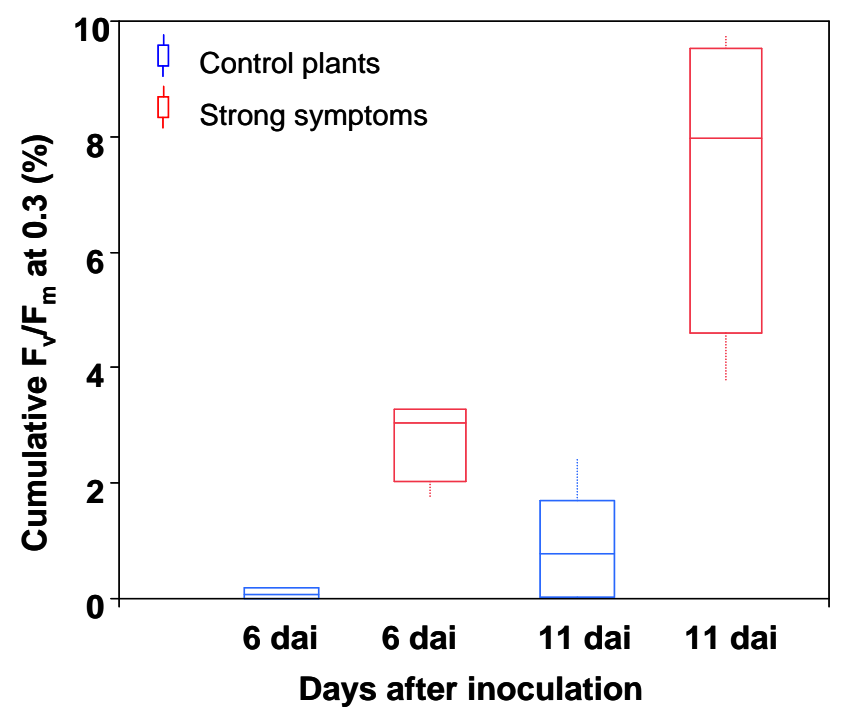

Field application of CFA imaging yielded in more variable differentiation of ears according to the severity of disease than laboratory studies (Figure 4). Discrepancy between visual inspection and CFA was more pronounced at low infection (sod < 30\%). Nevertheless, ears with medium (40-50\%) and high (70-80\% and 90\%, respectively) sod could easily be identified. Overall, correlation between the cumulative $\mathrm{F}_{\mathrm{v}} / \mathrm{F}_{\mathrm{m}}$ and sod was high in this experiment yielding a coefficient of determination of 0.658 (Figure 4).

Figure 4. Correlation between the cumulative $F_{v} / F_{m}$ at 0.3 and the severity of disease obtained from visual rating (circles: 23 June 2009, triangles: 24 June 2009).

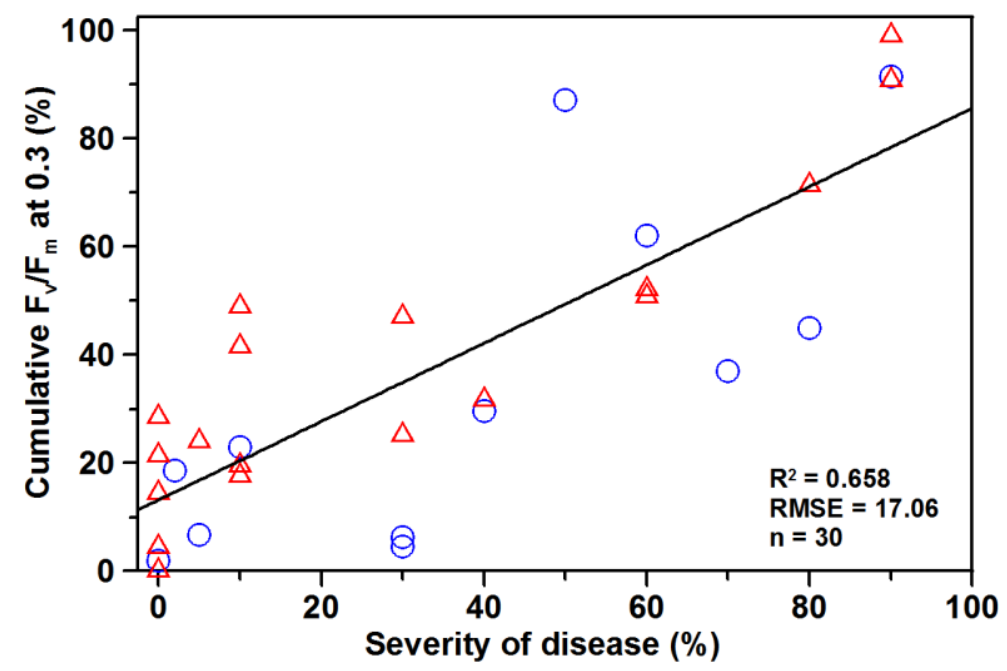




\subsection{Hyperspectral Measurements}

Time series experiments showed that differentiation by hyperspectral imaging was most effective at $14 \pm 2$ dai (Figure 5, dai 16 shown). It was less effective and, hence, results less reliable soon after infection and, again, after the beginning of maturation $c a$. four weeks after infection.

Figure 5. Samples of classification results using SAM classification (green: healthy classified tissues, red: diseased classified tissues). Upper row: infected ears, lower row: controls).

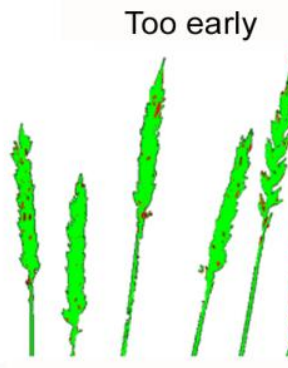

infected (12): 8 dai

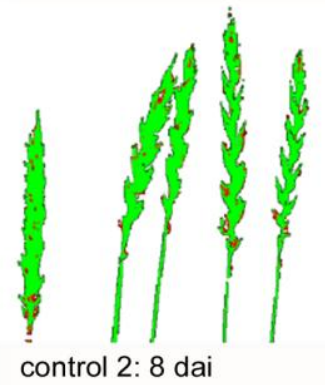

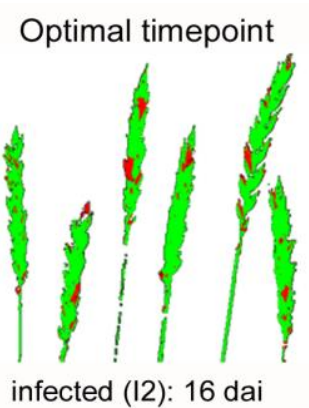

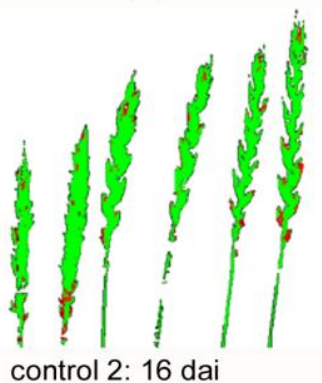

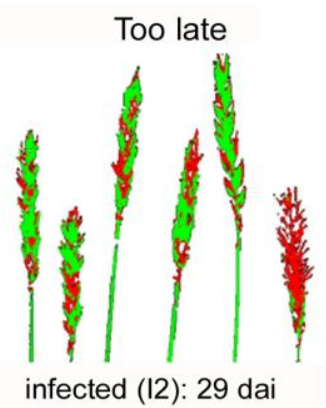

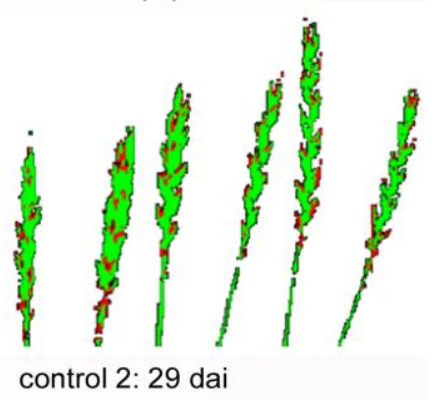

Results of classification of the levels of infection, obtained from hyperspectral imaging by the SAM algorithm, reflected those of the visual rating [Figure 6(a)].

Figure 6. (a) Results SAM-based classification of infected and control ears $(n=12)$ in comparison with the severity of disease obtained by visual rating during the course of infection development. (b) Proportion of pixels classified as infected and those unclassified by the SAM algorithm in comparison with the severity of disease obtained by visual rating $(n=6)$. Results of healthy ears are not shown.
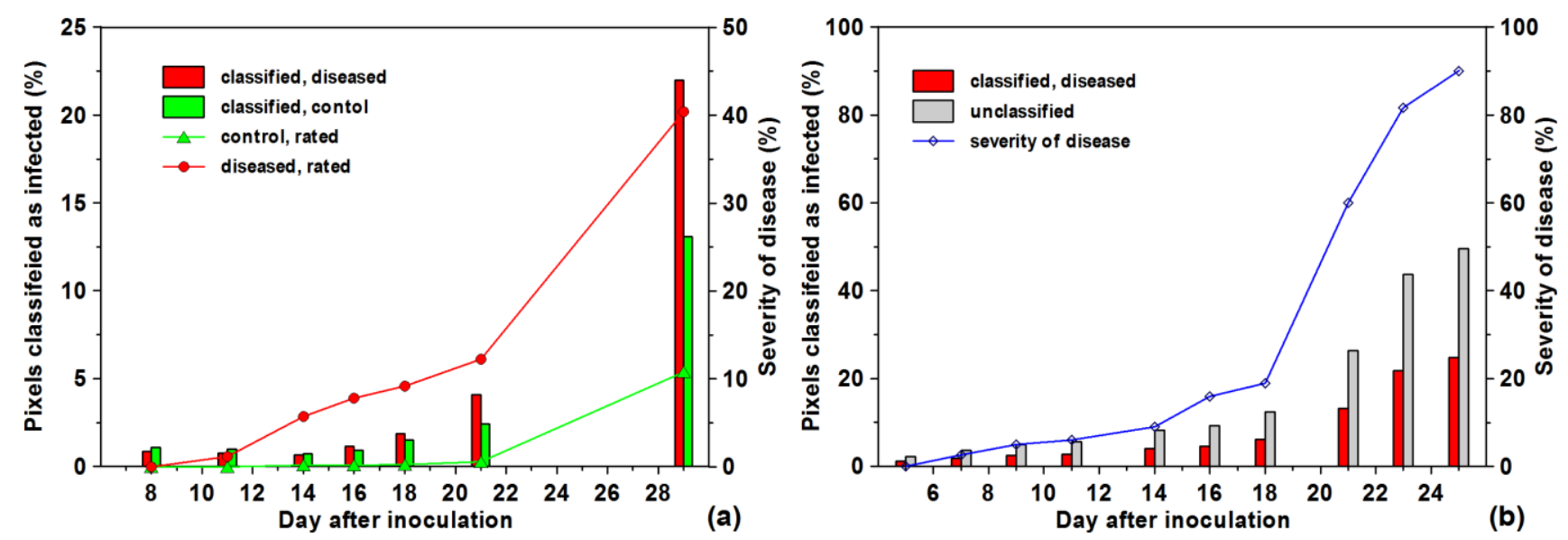
For this comparison, proportions of the whole ears, classified as diseased, were related to the total number of classified pixels (healthy + diseased, see Figure 5). During early infection, starting from $\mathrm{BBCH}$-stage 75, the sod obtained by spectral classification was always lower than that rated visually; it became closer at the beginning of ripening. Head blight was well separated from healthy tissues after the onset of ripening (from $\mathrm{BBCH} 81$, dai 21). However, at this stage, pixels, which previously were classified as healthy, were now increasingly ascribed as unclassified [Figure 6(b)]. Generally, results of the SAM evaluation algorithm applied to hyperspectral image analysis were highly correlated $\left(\mathrm{R}^{2}=0.964\right)$ with visually evaluated sod (data not shown). In all cases, the quality of classification strongly depended on the appropriate setting of ROIs. For this purpose, it is necessary to inspect the respective region of the image at the highest resolution.

\subsection{Effects of Ear Development on Quality of Head-Blight Detection}

The presented results indicate that during early ear development (starting from BBCH-stage 75), initial symptoms of infection can be eye-detected at 7 dai (Figure 7).

Figure 7. Time bar for the detection of head blight under indoor conditions.

first symptoms

rating

after 7 days

\begin{tabular}{|c|c|c|}
\hline & $\begin{array}{l}\text { hyperspectral } \\
\text { imaging }\end{array}$ & \\
\hline & BBCH 75/77 & \\
\hline inoculation & $\begin{array}{l}F_{v} / F_{m} \\
\text { measurement }\end{array}$ & $\begin{array}{l}F_{v} / F_{m} \\
\text { measurement }\end{array}$ \\
\hline day 0 & $\begin{array}{l}\text { first fixed date } \\
\text { after } 6 \text { days }\end{array}$ & $\begin{array}{l}\text { second fixed date } \\
\text { after } 11 \text { days }\end{array}$ \\
\hline BBCH 65 & BBCH 71 & BBCH 77 \\
\hline
\end{tabular}

no reliable detection

rating: healthy and

diseased ears

undistinguishable from

each other

incorrect hyperspectral and

$F_{v} / F_{m}$ - measurement

\section{maturity}

up to 15 th day

BBCH 81

Supervised classification of hyperspectral images in visual range identified first head blight symptoms at the same time. Most efficient classification of Fusarium-affected ears was possible in the $\mathrm{BBCH}$-stage 75 to 77 . With progressing maturation, the number of ears that could not be classified correctly largely increased. However, these limitations were also partially valid for CFA imaging. On the other hand, $\mathrm{F}_{\mathrm{v}} / \mathrm{F}_{\mathrm{m}}$-measurements detect symptoms of infection of ears earlier than visual rating and 
hyperspectral imaging. In general, highest accuracy of detection of Fusarium infection may be achieved if two successive measurement dates were performed at the growth stage "medium milk" (grain content is milky, BBCH 71-77; dai 6 to 11).

\section{Discussion}

To the best of our knowledge, this is the first investigation on the combined application of chlorophyll fluorescence and hyperspectral imaging for the early in vivo detection of head blight disease in winter wheat. It could be convincingly shown that both methods can indentify Fusarium infection of wheat ears non-invasively and with high reliability at a very early stage of disease.

Based on the physiology of photosynthesis, chlorophyll fluorescence imaging allowed detection of the initial phase of tissue damage. After the penetration of kernels by the mycelia, there are distinct cellular changes such as degeneration of cytoplasm and cell organelles, decomposition of the host's cell walls and deposition of material in vessel walls of the diseased ears [34]. Infection may lead to a complete inhibition of the metabolic activity, including a pronounced disturbance of photosynthetic performance. This can be easily identified by a rapid decline in the photochemical efficiency in the infected ears [24], even before visible chlorophyll degradation occurs.

Chlorophyll fluorescence imaging is a well-established effective tool to comprehensively assess the development and the effects of bacterial, fungal and viral infections on leaves of many crop plants (e.g., $[8,23,35])$. One topic of this study was to optimize both application of this technique and analysis of obtained results for rapid and early detection of head blight on wheat ears in laboratory and in field. To establish the level of infection of intact ears, the potential maximum photochemical efficiency of PSII $\left(F_{v} / F_{m}\right)$ was applied. In contrast to the analysis of absolute $F_{v} / F_{m}$ values, the statistical evaluation of its relative distribution in the entire image provided a successful approach for this purpose. The broadening of the overall distribution of $\mathrm{F}_{\mathrm{v}} / \mathrm{F}_{\mathrm{m}}$ with developing infection closely reflected the increased number of diseased kernels per ear.

The analysis of the cumulative $\mathrm{F}_{\mathrm{v}} / \mathrm{F}_{\mathrm{m}}$ allows an accurate evaluation of the changed distribution pattern. Considering a cumulative percentage at 0.3 as the differentiation threshold, levels of infection can be differentiated in 10\%-steps. Hence, even from the sixth dai, infected and control plants could be effectively separated in laboratory experiments.

Using this approach, it could be shown that the fungi seriously affect photosynthetic performance and, thus, chlorophyll fluorescence at an early stage. This has also been reported by $[8,36]$ for leaf pathogens. For instance, [8] found reduced $\mathrm{F}_{\mathrm{v}} / \mathrm{F}_{0}$-values two to three days before leaf rust and powdery mildew infection became visible on leaves of winter wheat.

In several studies, other image analysis approaches have been applied. Investigating yellow rust infection on wheat, [5] recorded fluorescence images at 550 and $690 \mathrm{~nm}$. From the relative signal intensities at these two wavelengths, the authors built a disease index $\left(f_{G}\right)$ and defined pixels exceeding the $f_{G}$ value of 0.65 as "diseased". For each leaf of Tulip Breaking Virus (TBV)-infected plants, [37] calculated mean and standard deviation in photochemical efficiency classes (0-1) of fluorescence images. With this procedure, they got higher error rates $(31-46 \%)$ than found by [5] or obtained in the present study. Classifying infected or healthy ears by the cumulative $F_{v} / F_{m}$ at 0.3 , as used in this study, is the fastest method of analysis. 
The current techniques of chlorophyll fluorescence imaging for identification of head blight in the field certainly need improvement. Due to their complex physiological nature [16], the fluorescence signals directly depend on the prevailing photosynthetic photon flux density. Hence, fluctuating light and direct exposure to sunlight must be avoided during measurements. Furthermore, before measurement of $F_{0}$ and $F_{m}$, plants need to be dark-adapted [5,17]. As shown in this study, these requirements can certainly be achieved.

Although the applied measuring system was developed for use in the laboratory, a suitable $\left(\mathrm{R}^{2}=0.658\right)$ correlation between fluorescence analysis and visible head blight inspection has been obtained under field conditions. The reduction of correlation quality may be due to the high subjectivity of visual rating. The scale applied for visual rating had a step size of $10 \%$; therefore, the absolute rating error would be $10 \%$ in the worst case. Methodological problems with the FluorCam measurements could not be entirely excluded but can be largely minimised by proper handling of the system.

Furthermore, movement of ears, induced by strong wind during recording of the sequences of $\mathrm{F}_{0}$ and Fm images, may result in non-overlaying frames of these two parameters. Therefore, overall recording time was reduced to $2 \mathrm{~s}$. Nevertheless, peripheral areas of ears, which were influenced by wind, may have incorrectly low $\mathrm{F}_{\mathrm{v}} / \mathrm{F}_{\mathrm{m}}$. However, the resulting poorly observable marginal regions at the border area of the ROIs may be excluded from further data analysis. Also, incomplete, uneven shading in the measuring box occasionally provide another problem, leading to an overestimated basic fluorescence and, hence, erroneously low $\mathrm{F}_{\mathrm{v}}$.

Elimination of all identified outliers reduced the amount of analysable ears by one third. As a consequence, the degree of determination of the correlation between fluorescence analysis and visible disease inspection rose to $\mathrm{R}^{2}=0.80$. This clearly indicates the high potential of chlorophyll fluorescence imaging for non-invasive disease detection after further improvement of measuring technique and protocols.

In case of hyperspectral imaging, the SAM classification algorithm used resulted in good and reliable detection of diseased ears. According to [32] SAM has a great potential for analysis in multi- and hyperspectral imaging. Nevertheless, the best classification method always depends on the complexity of the initial sets of data. To distinguish between Fusarium-infected and non-infected wheat ears, [38] evaluated RGB images with only three available channels and achieved better classification results by applying the Maximum-Likelihood-Method compared to the application of SAM. With increasing spectral information, other classification methods such as SAM [39], k-Nearest Neighbour, Decision Tree or Support Vector Machines [40] are certainly indispensable. However, basically improved imaging techniques, which allow reproducible and reliable data recording, may represent a necessary first step to optimize and, most important, automatize disease detection.

In general, hyperspectral images have a much higher information density than RGB. Hyperspectral image analysis is based on the entire spectral range investigated and it not only refers to three colour channels. Using spectral images (400-750 nm), [41] clearly separated different ripening stages of tomatoes, whereas application of RGB-images was not successful. In addition, the use of distinct ratios of different wavelengths for disease control and quality analysis has been widely reported [42-44]. In this context, to apply spectral imaging under field conditions, data gained by hyperspectral systems may be used to extract relevant wavelength ranges for rapid multispectral devices. 
An important problem with the disease classification by spectral imaging is the choice of the correct stage of development; otherwise the results may be inaccurate. If the measurements start too early, floral residues (anthers) and sterile ears caused by growth disorders are classified as diseased. Hence, in initial phases of the present studies, the low level of infection (ca. 3\%) classified by SAM on healthy ears (see Figure 6) was not based on head blight but reflected developmental disorders such as barren middle ears or tips. This means that damage other than that caused by Fusarium, were inevitably classified as diseased. Both types of damages could not be readily distinguished.

Classification results could, in some cases, be improved by either choosing different angle's radian specific to the respective object classes or by manually adjusting the angle's radian to lower values, e.g., to 0.05 . However, such variations showed to be advantageous only for ears in the BBCH stages 89 , because it also decreased the total number of classified pixel.

With the incipient ripeness, the spectra of healthy and diseased ears become more similar, which, again, leads to an increasing misclassification. Unclassified pixels clearly reflect the progressing degradation of chlorophyll during maturation, which occurred in healthy ears without the distinct spectral signature of infected kernels. In the classification procedure applied, such a class has not been specified but will be a next step of optimization. This has to be verified on the control plants which were free of a Fusarium infection.

Both methods investigated here are suitable for the detection of head blight. However, the next step to improve the accuracy of classification should be the dynamic combination of both methods and the addition of form and spot parameters, as proposed by [37]. A highly accurate classification is very important, because minimal levels of infection can lead to a contamination of major harvest lots with the poisonous mycotoxins of the Fusarium-fungi.

\section{Conclusions}

Laboratory as well as in-field measurements were performed to investigate the applicability of chlorophyll fluorescence and hyperspectral imaging for the detection of head blight. Under laboratory conditions, chlorophyll fluorescence imaging detects even very low levels of infection ( $\mathrm{ca}$. 5\%) as early as 6 dai; visual classification is only possible beginning from 7 dai.

One single measurement enables a distinction between infected and healthy ears, provided the disease is sufficiently strong. However, two measurement dates are recommended to reliably detect even a minimal infestation and to eliminate possible errors of measurement. By the use of the cumulative Fv/Fm threshold of 0.3 , the severities of infection can be detected with an accuracy of $10 \%$ under laboratory conditions. Under field conditions a differentiation between low (0-10\%), medium (40-50\%) and high (70-80\% and 90\%, respectively) level of infection can also be described with a linear model $\left(\mathrm{R}^{2}=0.658\right.$, RMSE $\left.=17 \%\right)$. Yet, the accuracy may rise up to $80 \%$ after data pre-processing including the elimination of outliers.

The application of the SAM evaluation algorithm yielded relatively good classification results. Nevertheless, the number of unclassified pixels increased during ear development.

The correct growth stage for spectral measurements and classification is therefore very important. From the $\mathrm{BBCH}$-stage 81 (beginning of ripening) on, a distinction between healthy and diseased ears by the methods discussed above is limited. 


\section{Acknowledgements}

This study is a part of the ProSenso.net ${ }^{2}$ research project "Sensor based technologies and integrated assessment models in the food production chains", which is financially supported by the German Federal Ministry of Education and Research (BMBF 0339992). We would like to thank H. Beuche and J. Intreß for their support and instruction on the laboratory hyperspectral device and B. Rodemann from the Julius-Kühn-Institute, Braunschweig, for providing the plant material.

\section{References}

1. Miedaner, T.; Schneider, B.; Geiger, H.H. Deoxynivalenol (DON) content and fusarium head blight resistance in segregating populations of winter rye and winter wheat. Crop Sci. 2003, 43, 519-526.

2. Thate, A.; Schumann, S.; Hanschmann, G.; Urban, Y. Auftreten von Ährenfusariosen und Mykotoxinen in Sachsen und Ergebnisse der Vor- und Nachernteuntersuchungen von Winterweizen 2007 (in German). Nachrichtenbl. Dtsch. Pflanzenschutzdienst 2008, 60, 117.

3. Oerke, E.C.; Steiner, U.; Dehne, H.W.; Lindenthal, M. Thermal imaging of cucumber leaves affected by downy mildew and environmental conditions. J. Exp. Botany 2006, 57, 2121-2132.

4. Castro, K.L.; Sanchez-Azofeifa, G.A. Changes in spectral properties, chlorophyll content and internal mesophyll structure of senescing Populus balsamifera and Populus tremuloides leaves. Sensors 2008, 8, 51-69.

5. Bravo, C.; Moshou, D.; Oberti, R.; West, J.; McCartney, A.; Bodria, L.; Ramon, H. Foliar disease detection in the field using optical sensor fusion. Agr. Eng. Int. CIGR J. Sci. Res. Dev. 2004, 6, Manuscript FP 04008.

6. Larsolle, A.; Muhammed, H.H. Measuring crop status using multivariate analysis of hyperspectral field reflectance with application to disease severity and plant density. Precis. Agr. 2007, 8, 37-47.

7. Meroni, M.; Rossini, M.; Picchi, V.; Panigada, C.; Cogliati, S.; Nali, C.; Colombo, R. Assessing steady-state fluorescence and PRI from hyperspectral proximal sensing as early indicators of plant stress: The case of ozone exposure. Sensors 2008, 8, 1740-1754.

8. Kuckenberg, J.; Tartachnyk, I.; Noga, G. Temporal and spatial changes of chlorophyll fluorescence as a basis for early and precise detection of leaf rust and powdery mildew infections in wheat leaves. Precis. Agr. 2009, 10, 34-44.

9. Delwiche, S.R. Classification of scab- and other mold-damaged wheat kernels by near-infrared reflectance spectroscopy. Trans. ASAE 2003, 46, 731-738.

10. Sims, D.A.; Gamon, J.A. Estimation of vegetation water content and photosynthetic tissue area from spectral reflectance: A comparison of indices based on liquid water and chlorophyll absorption features. Remote Sens. Environ. 2003, 84, 526-537.

11. Polischuk, V.P.; Shadchina, T.M.; Kompanetz, T.I.; Budzanivskaya, I.G.; Boyko, A.L.; Sozinov, A.A. Changes in reflectance spectrum characteristic of Nicotiana debneyi plant under the influence of viral infection. Arch. Phytopath. Pflanzenschutz 1997, 31, 115-119.

12. Aldea, M.; Frank, T.D.; DeLucia, E.H. A method for quantitative analysis of spatially variable physiological processes across leaf surfaces. Photosynth. Res. 2006, 90, 161-172. 
13. Franke, J.; Menz, G.; Oerke, E.C.; Rascher, U. Comparison of multi- and hyperspectral imaging data of leaf rust infected wheat plants. Proc. SPIE 2005, doi:10.1117/12.626531.

14. Lorenzen, B.; Jensen, A. Changes in leaf spectral properties induced in barley by cereal powdery mildew. Remote Sens. Environ. 1989, 27, 201-209.

15. Muhammed, H.H.; Larsolle, A. Feature vector based analysis of hyperspectral crop reflectance data for discrimination and quantification of fungal disease severity in wheat. Biosyst. Eng. 2003, $86,125-134$.

16. Maxwell, K.; Johnson, G.N. Chlorophyll fluorescence-A practical guide. J. Exp. Botany 2000, 51, 659-668.

17. von Willert, D.J.; Matyssek, R.; Herppich, W.B. Experimentelle Pflanzenökologie, Grundlagen und Anwendungen (in German); Georg Thieme Verlag: Stuttgart, Germany, 1995.

18. Baker, N.R.; Rosenqvist, E. Application of chlorophyll fluorescence can improve crop production strategies: An examination of future possibilities. J. Exp. Botany 2004, 55, 1607-1621.

19. Quilliam, R.S.; Swarbrick, P.J.; Scholes, J.D.; Rolfe, S.A. Imaging photosynthesis in wounded leaves of Arabidopsis thaliana. J. Exp. Botany 2006, 57, 55-69.

20. Herppich, W.B. Application potential of chlorophyll fluorescence imaging analysis in horticultural research. In Proceedings of the 6th International Symposium: Fruit, Nut and Vegetable Production Engineering, Potsdam, Germany, 11-19 September 2001; pp. 609-614.

21. Herppich, W.B.; Linke, M.; Landahl, S.; Gzik, A. Preharvest and postharvest responses of radish to reduced water supply during growth. Acta Horticult. 2001, 553, 89-90.

22. Kuckenberg, J.; Tartachnyk, I.; Schmitz-Eiberger, M.; Noga, G. Early detection of leaf rust and powdery mildew infections on wheat leaves by PAM fluorescence imaging. Precis. Agr. 2007, 8, 515-521.

23. Daley, P.F. Chlorophyll fluorescence analysis and imaging in plant stress and disease. Can. J. Plant Patho. 1995, 17, 167-173.

24. Bauriegel, E.; Giebel, A.; Herppich, W.B. Rapid Fusarium head blight detection on winter wheat ears using chlorophyll fluorescence imaging. J. Appl. Botany Food Quality 2010, 83, 196-203.

25. Bauriegel, E.; Giebel, A.; Geyer, M.; Schmidt, U.; Herppich, W.B. Early detection of Fusarium infection in wheat using hyper-spectral imaging. Comput. Elect. Agr. 2011, in press, doi:10.1016/j.compag.2010.12.006.

26. Lancashire, P.D.; Bleiholder, H.; Vandenboom, T.; Langeluddeke, P.; Strauss, R.; Weber, E.; Witzenberger, A. A uniform decimal code for growth-stages of crops and weeds. Ann. Appl. Biol. 1991, 119, 561-601.

27. Walther, U.; Flath, K.; Moll, E.; Prochnow, J.; Sachs, E. Methodische Anleitung zur Bewertung der partiellen Resistenz von Sorten bzw. Linien unter Berücksichtigung epidemiologischer Aspekte (in German). Mitt. Biol. Bundesanst. Land- Forstwirtsch. 2000, 374, 9-25.

28. Nedbal, L.; Trtìlek, M.; Herppich, W.B. Methods and equipment for fluorescence imaging on plant material. Bornimer Agrartechn. Ber. 2000, 25, 127-135.

29. Schlüter, O.; Förster, J.; Geyer, M.; Knorr, D.; Herppich, W.B. Characterization of high-hydrostatic-pressure effects on fresh produce using chlorophyll fluorescence image analysis. Food Bioprocess Tech. 2009, 2, 291-299.

30. Krause, G.H.; Weis, E. Chlorophyll fluorescence and photosynthesis: the basics. Ann. Rev. Plant Phys. Plant Mol. Biol.1991, 42, 313-349. 
31. ENVI User's Guide; Research Systems, Inc.: Boulder, CO, USA, 2005; pp. 1-1202.

32. Petropoulos, G.P.; Vadrevu, K.P.; Xanthopoulos, G.; Karantounias, G.; Scholze, M. A comparison of spectral angle mapper and artificial neural network classifiers combined with Landsat TM imagery analysis for obtaining burnt area mapping. Sensors 2010, 10, 1967-1985.

33. University of Texas at Austin CSR. Analysis of Hyperspectral Imagery. Available online: www.csr.utexas.edu/projects/rs/hrs/analysis.html (accessed on 10 July 2010).

34. Kang, Z.; Buchenauer, H. A cytological and ultrastructural study on the infection process of Fusarium culmorum on wheat spikes. Mycol. Res. 2000, 104, 1083-1093.

35. Scholes, J.D.; Rolfe, S.A. Photosynthesis in localised regions of oat leaves infected with crown rust (Puccinia coronata): Quantitative imaging of chlorophyll fluorescence. Planta 1996, 199, 573-582.

36. Förschler, A.; Portz, C.; Steiner, U.; Schmitz-Eiberger, M.; Noga, G. Minderung von pilzlichem Pathogenbefall im organischen Obstbau durch Zufuhr von Antioxidantien (in German); Landwirtschaftliche Fakultät der Universität Bonn, Schriftenreihe des Lehr- und Forschungsschwerpunktes USL 113: Bonn, Germany, 2003; pp. 1-41.

37. Polder, G.; van der Heijden, G.W.A.M.; van Doorn, J.; van der Schoor, R.; Baltissen, A.H.M.C. Detection of the tulip breaking virus (TBV) in tulip using spectral and vision sensors. In Proceedings of the 7th European Conference on Precision Agriculture, Wageningen, The Netherlands, 6-8 July 2009; pp. 219-227.

38. Hehmke, M.; Dammer, K.H.; Herppich, W.B.; Hellebrand, J.; Beuche, H.; Rodemann, B. Digital image analysis for detection of head blight (Fusarium spp.) in winter wheat. In Proceedings of the International Symposium: Agricultural Field Trials-Today and Tomorrow, Stuttgart, Germany, 8-10 October 2007; pp. 56-61.

39. ENVI Tutorial. Selected Hyperspectral Methods. Available online: www.creaso.com (accessed on 7 January 2010).

40. Weis, M.; Rumpf, T.; Gerhards, R.; Plümer, L. Comparison of different classification algorithms for weed detection from images based on shape parameters. Bornimer Agrartechn. Ber. 2009, 69, 53-64.

41. Polder, G.; van der Heijden, G.W.A.M.; Young, I.T. Spectral image analysis for measuring ripeness of tomatoes. Trans. ASAE 2002, 45, 1155-1161.

42. Gitelson, A.A.; Gritz, Y.; Merzlyak, M.N. Relationships between leaf chlorophyll content and spectral reflectance and algorithms for non-destructive chlorophyll assessment in higher plant leaves. J. Plant Phys. 2003, 160, 271-282.

43. Lichtenthaler, H.K. Spektroskopische Eigenschaften von Pflanzen und ihre Nutzung zur Fernerkundung der Vegetation (in German). Fridericiana 1994, 49, 25-45.

44. Nicotra, A.B.; Hofmann, M.; Siebke, K.; Ball, M.C. Spatial patterning of pigmentation in evergreen leaves in response to freezing stress. Plant Cell Environ. 2003, 26, 1893-1904.

(C) 2011 by the authors; licensee MDPI, Basel, Switzerland. This article is an open access article distributed under the terms and conditions of the Creative Commons Attribution license (http://creativecommons.org/licenses/by/3.0/). 